\title{
基于表面增强拉曼光谱的食品中二氧化硫残留的 快速检测方法
}

张琳 ${ }^{1}$, 曾勇明 ${ }^{2,3}$, 赵建 ${ }^{\text {军 }}{ }^{1}$, 陈宏炬 ${ }^{4}$, 孔景临 ${ }^{1}$, 陈启振 ${ }^{2,3}$, 林惠真 ${ }^{4}$, 田中群 ${ }^{4}$, 刘国坤2*

1. 国民核生化灾害防护国家重点实验室, 北京 102205

2. 厦门大学环境与生态学院, 近海海洋环境科学国家重点实验室, 厦门 361102

3. 厦门市普识纳米科技有限公司, 厦门 361021

4. 厦门大学化学化工学院, 厦门 361005

*通讯作者, E-mail: guokunliu@xmu.edu.cn

收稿日期: 2016-11-10; 接受日期: 2017-02-13; 网络版发表日期: 2017-04-18

国家自然科学基金(编号: 21473140)、福建省高校产学合作项目(编号: 2016Y4012)和中央高校基本科研业务费专项资金(编号: 2072016011) 资助项目

摘要基于表面增强拉曼光谱(SERS)的指纹图谱的高能量分辨率, 以位于 $630 \mathrm{~cm}^{-1}$ 亚硫酸根的特征峰为 定性和定量依据, 本文开展了食品中滥用和非法添加二氧化硫的直接检测研究: 一种检测流程实现了不同食 品基质中二氧化硫的高灵敏检测, 最低检出浓度达 $1 \mathrm{mg} \mathrm{kg}^{-1}$ 的水平. 针对实际样本的传统蒸馏法前处理流程 的一些不足以及SERS检测的指纹图谱优势, 本文进行了三方面显著改进: (1) 碳酸钻和沸石取代氮气以提高 二氧化硫的挥发提取效率; (2) 草酸取代盐酸酸化蒸馏溶液, 以有效避免挥发性强酸对操作环境的危害; (3) 氢氧化钠溶液代替乙酸铅溶液作为吸收液, 以防止二次污染。环境友好、灵敏度高和不受基质干扰等特点 使得本方法有望取代经典蒸馏法和比色法, 用于食品中非法添加二氧化硫类添加剂的快速高效節查.

关键词表面增强拉曼光谱, 快速篮查, 环境友好, 高灵敏

\section{1 引言}

为了促进人们的购买欲, 提高食品的商品价值, 食 品加工过程中常会使用多种食品添加剂. 其中, 二氧 化硫能使食品保持鲜艳色泽的同时防止食品褐变和 防腐, 所以, 可解离生成二氧化硫的亚硫酸钠和焦亚 硫酸钠(钾)等物质是食品加工过程中常用的漂白剂和 防腐剂. 少量二氧化硫进入体内后可通过正常机体代
谢解毒后由尿排出体外, 但是, 如果摄入量严重超标, 将引发恶心、呕吐及影响钙吸收等危害人体健康的 不良影响. GB 2760-2011《食品安全国家标准食品添 加剂使用标准》明确规定了二氧化硫的使用范围和 限量. 然而, 近年来, 不仅超限量使用二氧化硫的食品 安全案例屡见不鲜, 甚至发生了多起美白土豆、光鲜 生姜和鲜肉等非法添加事件.例如, 2016年7月22日, 香港食物环境卫生署食物安全中心在新鲜牛肉样本

引用格式: 张琳, 曾勇明, 赵建军, 陈宏炬, 孔景临, 陈启振, 林惠真, 田中群, 刘国坤. 基于表面增强拉曼光谱的食品中二氧化硫残留的快速检测方 法. 中国科学: 化学, 2017, 47: 794-800

Zhang L, Zeng Y, Zhao J, Chen H, Kong J, Chen Q, Lin H, Tian Z, Liu G. Developing fast laboratory screening platform for sulfate dioxide in food by surface-enhanced Raman spectroscopy. Sci Sin Chim, 2017, 47: 794-800, doi: 10.1360/N032016-00197 
中检出非法添加的二氧化硫.

食品中二氧化硫检测的经典方法是蒸馏法(酸性 条件下进行碘滴定)和盐酸副玫瑰苯胺比色法. 虽然这 两种方法已作为国标GB/T5009.34-2003使用, 但方法 所存在的前处理复杂、试剂污染环境、实验操作繁 兄且选择性不佳等缺点使得研究者们不断开发基于 色谱(液相色谱、气相色谱和高效液相色谱(HPLC))、 离子色谱、原子吸收光谱、生物芯片、电化学、表 面增强拉曼光谱等多种技术的检测方法, 并结合微流 控和流动注射等技术, 以提高二氧化硫检测的灵敏度 和选择性, 目前已经取得了良好的测试结果 ${ }^{[1 \sim 2]}$. 然而, 这些方法, 要么仪器昂贵、体积大、操作复杂, 要么 前处理过程费时费力, 很难作为一种实验室快速篮查 方法以应对实际检测工作中的大量检测样本. 因此, 有必要发展一种高灵敏、准确可靠且简单的实验室 快速篮查方法, 提高相关职能部门的应急能力.

近年来, 具有分子指纹信息和痕量物种检测能力 的表面增强拉曼光谱(SERS)技术的实际应用开始受 到广泛关注, 已成功用于食品安全领域痕量有害物质 的高灵敏检测, 如奶粉中三聚氧胺和橙子表皮的有机 磷农药等. 然而, 大部分工作的重点要么注重于SERS 基底对痕量标准物质的增强性能优化,与实际体系间 差异显著; 或以实际样本为检测对象, 但采用的是常规 实验室前处理方案, 操作复杂耗时. 只有结合拉曼光 谱的指纹图谱特点, 发展高效、快速、简便和可靠的 前处理技术, 并设计合适的SERS基底, 方能将SERS光 谱技术发展为可在食品安全领域实际应用的新技术.

SERS技术已成功用于检测葡萄酒中的二氧化硫， 检测水平和实验室常规技术相当, 然而该方法未能进 一步拓展到各类可能含有二氧化硫类添加剂的食品, 而且使用强腐蚀性的硫酸作为酸性介质 ${ }^{[10]}$, 不利于推 广. 因此, 针对二氧化硫滥用、非法添加屡见不鲜, 现 有快速检测方案尚显不足的现状, 本文发展了一种快 速SERS检测方案: 对传统蒸馏法进行改良, 以碳酸钲 和沸石取代氮气, 有效避免前处理中通氮气所需庞大 外围设备; 以草酸取代盐酸酸化蒸馏溶液, 显著降低 挥发性强酸对人体和环境的危害; 以氢氧化钠溶液代 替乙酸铅溶液作为吸收液, 消除重金属离子的引入和 二次污染. 所发展的方法成功实现了对滥用和非法添 加二氧化硫的海鲜干制品、土豆和生姜等生鲜食品 的快速有效检测. 该方法具有便捷、高灵敏和环境友
好等特点.

\section{2 实验部分}

\section{1 材料}

氯金酸 $\left(\mathrm{HAuCl}_{4}\right)$ 、柠檬酸钠、盐酸、草酸、硫 酸、磷酸和氢氧化钠等试验中所用试剂皆为分析纯 (上海国药, 中国), 鱼片等样本则采购自大型超市. 水 溶液皆用超纯水配置(Millipore).

\subsection{SERS增强活性的金纳米粒子的制备和表征}

利用经典的柠檬酸钠还原 $\mathrm{HAuCl}_{4}$ 的方法制备得 到粒径约为 $55 \mathrm{~nm}$ 的金纳米粒子 ${ }^{[13]}$, 该粒子由厦门市 普识纳米科技有限公司(中国)提供. 制备过程简介如 下: $1.2 \mathrm{~mL} 1 \%$ 柠檬酸钠迅速加入到含有 $1 \mathrm{mmol} \mathrm{L}^{-1}$ $\mathrm{HAuCl}_{4}$ 的 $100 \mathrm{~mL}$ 沸水溶液中, 并继续剧烈摚拌 $40 \mathrm{~min}$, 从而得到红色粒径约在 $55 \mathrm{~nm}, \mathrm{SPR}$ 吸收峰在 $537 \pm 1 \mathrm{~nm}$ 的金纳米溶胶.

\section{3 实际样本的前处理和SERS检测}

样本的提取预处理过程分为两步: (1) 称取一定 量的碳酸钻和沸石于 $100 \mathrm{~mL}$ 圆底烧瓶中, 加入 $30 \mathrm{~mL}$ 水溶解, 然后加入 $1 \mathrm{~g}$ 剪碎的样本和一定量的草酸溶 液. 用 $4 \mathrm{~mL} 10 \mathrm{mmol} \mathrm{L}^{-1} \mathrm{NaOH}$ 溶液收集蒸馏挥发出的 二氧化硫气体; (2) 加热样本, 当接收管的液体体积由 $4 \mathrm{~mL}$ 增加到 $6 \sim 7 \mathrm{~mL}$ 时, 停止加热. 整个前处理时间约 为 $30 \mathrm{~min}$. 需指出, 由于SERS的指纹图谱特点, 可有效 避开一些样本中的基质干扰, 如对于冰糖等样本, 将 样本溶解于水后即可进行SERS检测; 对于土豆和生 姜等样本, 可将金纳米溶胶滴加于样本表面进行检测, 而无需任何前处理.

SERS检测步骤如下: 取 $1.5 \mathrm{~mL}$ 纳米金以 $8000 \mathrm{r} / \mathrm{min}$ 离心 $5 \mathrm{~min}$, 取 $10 \mu \mathrm{L}$ 浓缩液与 $200 \mu \mathrm{L}$ 待测样本和 $10 \mu \mathrm{L}$ $1 \mathrm{~mol} \mathrm{~L}^{-1} \mathrm{NaCl}$ (促进胶体金团聚形成有效SERS热点) 混匀后开始拉曼测试, 谱图采集时间为5 10 s.

\section{4 拉曼光谱仪}

SERS谱图皆于B\&W TEK公司的iRaman拉曼光谱 仪(美国)上完成. 该仪器的激光波长为 $785 \mathrm{~nm}$, 功率约 为 $275 \mathrm{~mW}, 10$ 倍物镜下达到样本表面的激光光斑大小 约为 $80 \mu \mathrm{m}$. 


\section{3 结果与讨论}

\section{1 亚硫酸钠的SERS谱图}

图1给出溶于 $10 \mathrm{mmol} \mathrm{L}^{-1}$ 氢氧化钠溶液中不同 浓度亚硫酸钠的SERS 谱图, 与无特征谱峰的空白谱 图相比, $0.1 \mathrm{mg} \mathrm{kg}^{-1}$ 亚硫酸钠即可以明显观察到位于 $\sim 494$ 和 $630 \mathrm{~cm}^{-1}$ 的两个谱峰, 其峰强和亚硫酸钠浓 度呈正向跟随关系, 可指认为亚硫酸根特征峰 ${ }^{[14]}$, 分 别归属于 $\mathrm{SO}_{3}$ 基团的对称和非对称弯曲振动 ${ }^{[15]}$. 仔 细观察谱图特征可发现, 位于 $630 \mathrm{~cm}^{-1}$ 的非对称弯 曲振动的峰形较宽, 在 $609 \mathrm{~cm}^{-1}$ 附近有一个肩峰, 则 可能来自于亚硫酸氢根中 $\mathrm{SO}_{3}$ 基团的非对称弯曲振 动 ${ }^{[15]}$, 也不排除含有微量亚硫酸根生成的焦亚硫酸根 中 $\mathrm{SO}_{3}$ 基团的非对称弯曲振动的贡献 ${ }^{[15,16]}$. 609和 630 $\mathrm{cm}^{-1}$ 两个谱峰的相对强度的变化反映了检测体系中亚 硫酸钠和亚硫酸氢钠比例随亚硫酸钠浓度的变化趋 势 $[16]$

图1插图中给出位于 $630 \mathrm{~cm}^{-1}$ 谱峰的归一化强度 随亚硫酸根浓度增加呈现正向跟随关系的趋势: 空 白无信号, $<10 \mathrm{mg} \mathrm{kg}^{-1}$ 时, SERS信号迅速增强; 10 50 $\mathrm{mg} \mathrm{kg}^{-1}$ 区间, SERS信号增强缓慢, 继续提高浓度则 趋于饱和. 对于实际样本, 二氧化硫的使用需在 100 $\mathrm{mg} \mathrm{kg}^{-1}$ 以上方有防腐等效果. 因此, 在后续的样本加 标实验中, 选择了 $50 \mathrm{mg} \mathrm{kg}^{-1}$ 亚硫酸钠作为加标浓度, 以验证方法的可靠性.

\section{2 酸的选择}

国标中蒸馏法的基本原理是使用盐酸溶液与含 有二氧化硫(或亚硫酸钠、焦亚硫酸钠)样本混合, 反 应生成的亚硫酸在加热情况下分解为二氧化硫气体, 利用乙酸铅收集该气体再通过酸化等步骤检测样本 中二氧化硫(或亚硫酸钠、焦亚硫酸钠)的含量. 虽然 有不少工作提出了各种改进的方法, 但蒸馏和酸化都 基本采用挥发性的盐酸, 有些工作则改为使用不易挥 发但强腐蚀性的硫酸 ${ }^{[10]}$. 鉴于这两种酸的使用都会造 成环境污染和对人体的潜在危害, 本研究首先考察了 不同酸对二氧化硫提取效率的影响, 择优选取环境友 好的酸性溶液.

以加标 $50 \mathrm{mg} \mathrm{kg}^{-1}$ 亚硫酸钠的烤鱼片为测试样本, 以 $1 \mathrm{~g}$ 碳酸钙为鼓泡剂, 采用浓度为 $1 \mathrm{~mol} \mathrm{~L}^{-1}$ 的草酸、 盐酸、硫酸和磷酸分别进行蒸馏, 以氢氧化钠为提取

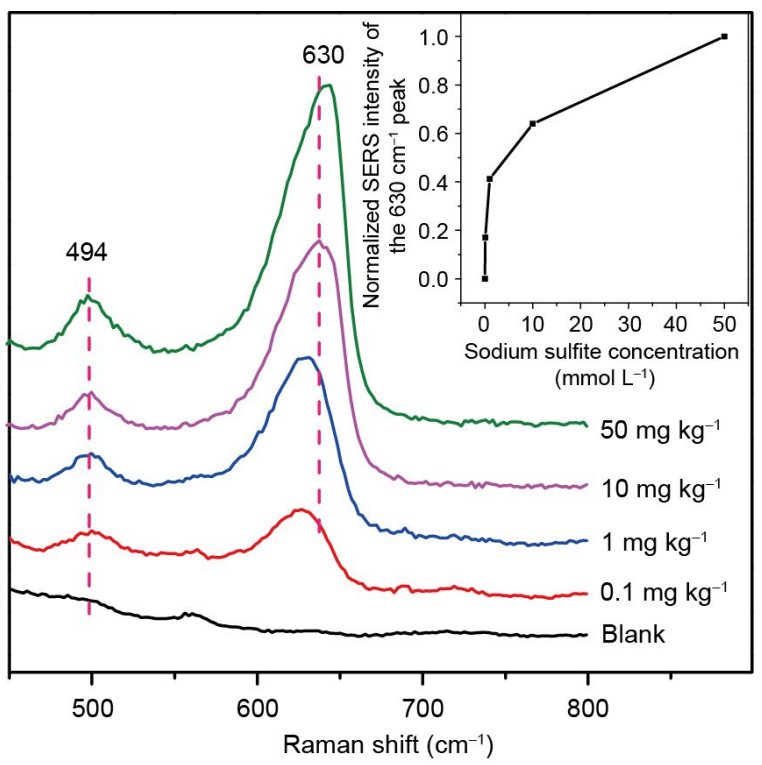

图 $110 \mathrm{mmol} \mathrm{L}^{-1}$ 氢氧化钠溶液中, 不同浓度亚硫酸的SERS 谱图, 插图为位于 $630 \mathrm{~cm}^{-1}$ 亚硫酸钠特征峰的归一化SERS 强度随其浓度的变化趋势(网络版彩图)

液, 提取二氧化硫 $30 \mathrm{~min}$ 后, 对所得提取液进行SERS 测试, 所得SERS 谱图如图2所示. 为更好地进行比较, 以醋酸蒸馏未加标样本得到的提取液的SERS 谱图作 为空白对照.

和标准品的空白相比, 提取液空白在 494和 $\sim 740 \mathrm{~cm}^{-1}$ 附近出现了两个谱峰, 在 $630 \mathrm{~cm}^{-1}$ 处有微 微隆起. 推测 $630 \mathrm{~cm}^{-1}$ 谱峰可能来自于样本中存在的 天然微量二氧化硫类物质的贡献; 494和 $740 \mathrm{~cm}^{-1}$ 谱 峰则可能来自于鱼片样本中易挥发性物质. 该物质的 提取效率受酸度影响明显, 硫酸介质中明显强于其他 酸性介质; 这两个谱峰在其他非鱼片样本体系中消失 (详见下文)也间接证明了来自于鱼片样本自身. 此外, 图2左上插图给出 $\mathrm{pH}$ 从9降低到7时, $50 \mathrm{mg} \mathrm{kg}^{-1}$ 亚硫酸 钠溶液的SERS 谱图变化, 可以发现, 随 $\mathrm{pH}$ 降低, 亚硫 酸钠的SERS谱图信噪比变差、强度减弱的同时, 494 $\mathrm{cm}^{-1}$ 谱峰的相对强度显著增强.

考虑到蒸馏提取亚硫酸钠引起提取液 $\mathrm{pH}$ 变化、 不同 $\mathrm{pH} \sim 494 \mathrm{~cm}^{-1}$ 谱峰强度的自身不稳定性以及来 自样品的干扰, 后续讨论中, 仅围绕位于 $630 \mathrm{~cm}^{-1}$ 的 亚硫酸根特征峰: 未加标鱼片样本在该处的极弱信号 与加标样信号间的显著差异, 说明实际样本的基质对 SERS检测无干扰, 即方法具有强定性能力和无假阳性 


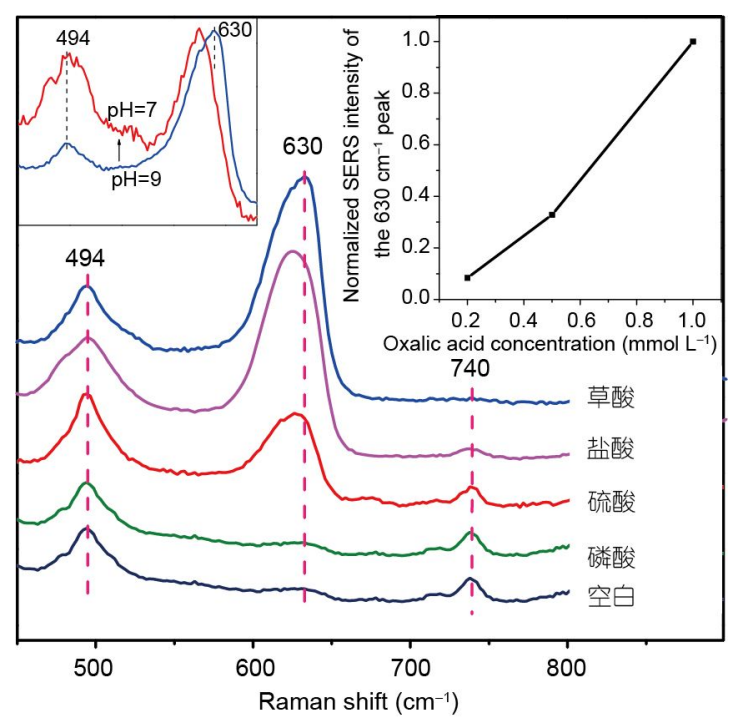

图 $21 \mathrm{~mol} \mathrm{~L}^{-1}$ 不同酸介质提取 $50 \mathrm{mg} \mathrm{kg}^{-1}$ 亚硫酸得到的 SERS谱图, 左上插图为 $\mathrm{pH} 7$ 和 9 时得到的SERS谱图; 右上插 图为位于 $630 \mathrm{~cm}^{-1}$ 亚硫酸钠特征峰归一化强度随草酸浓度 的变化趋势 (网络版彩图)

的优势.

与图 1中 $50 \mathrm{mg} \mathrm{kg}^{-1}$ 的亚硫酸钠标准品SERS 谱图 进行比对, 可以发现不同酸的提取回收率差异明显: 磷酸提取得到的SERS 谱图和空白几乎完全一致, 说 明酸性弱于亚硫酸的酸介质无提取效果; 硫酸一般 ( $18 \%$, 以 $630 \mathrm{~cm}^{-1}$ 特征峰强度计算), 盐酸( $\left.41 \%\right)$ 和 草酸( 45\%)都相对较好, 所有酸的提取回收率都不足 $50 \%$ 的可能原因在于: 在蒸馏过程中, 碳酸钙与酸反应 生成二氧化碳的副反应不可避免消耗了部分酸(见下 文). 提取液中加标 $0.1 \mathrm{~mol} \mathrm{~L}^{-1}$ 碳酸钠的对照实验未见 碳酸根的特征峰 ${ }^{[14]}$, 也表明碳酸的形成对亚硫酸根的 检测无干扰. 不同酸提取回收率的顺序和文献[10]报 道的有一定差异的原因可能在于, 文献中使用 $\mathrm{ZnO}$ 纳 米材料作为提取富集材料.

为什么作为弱酸, 草酸的提取效率反而高于硫酸 和盐酸等强酸? 初步推测可能有两个原因: (1) 区分效 应: 相比于亚硫酸, 碳酸为弱酸, 在硫酸或盐酸等强酸 存在下, 碳酸钻比亚硫酸钠更容易与强酸反应; (2) 拉 平效应: 草酸、亚硫酸和碳酸皆弱酸, 三者酸性差异 不显著, 相比强酸介质, 草酸与碳酸钙或亚硫酸根的 反应速率相差甚微. 两个因素协同作用下, 导致草酸 提取效率更佳.

相比于浓硫酸的强腐蚀性, 浓盐酸的强挥发性, 草
酸的性质温和便于实际操作. 此外, 根据图2插图所示 亚硫酸根位于 $630 \mathrm{~cm}^{-1}$ 的特征峰谱峰强度随草酸浓 度增加的变化趋势, 可以看到, 二氧化硫的提取效率 随草酸浓度增加而提高, 在接近草酸的饱和浓度的 1 $\mathrm{mol} \mathrm{L}^{-1}$ 时达到最强. 因此, 综合考虑, 以 $1 \mathrm{~mol} \mathrm{~L}^{-1}$ 草酸 作为酸性溶液提取食品中残留的二氧化硫.

\section{3 碳酸钻量和回流时间的控制}

在传统蒸馏法中, 为了提高蒸馏的效率, 主要采 取两种手段: 以氮气作为鼓泡剂和延长蒸馏时间. 引 入氮气可以降低二氧化硫在溶液中的溶解度, 从而提 高蒸馏效率, 但这需要额外装配一个氮气瓶和进气装 置. 考虑到碳酸钙在酸性条件下生成二氧化碳且其酸 性和亚硫酸相当, 二氧化碳是否可作为分气体降低二 氧化硫在溶液中的溶解度?

图3为不同碳酸钻添加量下所得到的亚硫酸根的 SERS信号, 考察了碳酸钻添加量对二氧化硫提取效率 的影响, 所得结果表明, 碳酸钙可以作为起泡剂, 促进 二氧化硫的生成; 碳酸钙量较少时, 提取效率很低, 可 能是因为在蒸馏初期, 大部分碳酸钻已转化为二氧化 碳而无法在蒸馏后期作为分气体以降低二氧化硫的 溶解度. 随着碳酸钻量的增加, 二氧化硫的提取效率 不断升高, $1 \mathrm{~g}$ 时达到最大. 虽然继续增加碳酸钻量将

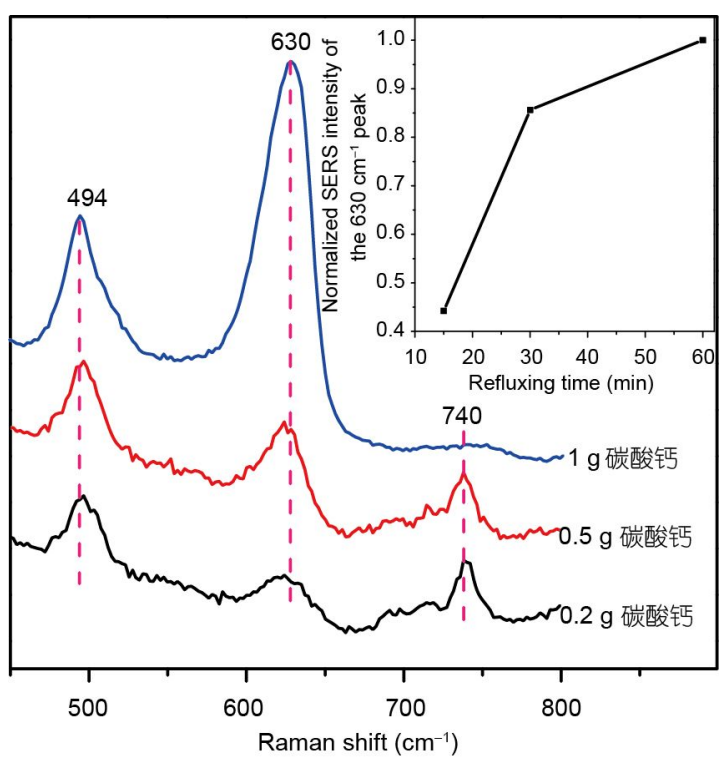

图 3 不同碳酸钻量下提取 $50 \mathrm{mg} \mathrm{kg}^{-1}$ 亚硫酸得到的SERS 谱图, 插图为 $1 \mathrm{~g}$ 碳酸钙量时, 位于 $630 \mathrm{~cm}^{-1}$ 亚硫酸钠特征峰 的归一化强度随蒸馏时间的变化趋势 (网络版彩图) 
进一步提高二氧化硫提取效率, 但同时需要消耗大量 草酸, 反而降低提取效率.

图3插图给出了蒸馏时间对提取效率的影响, 随 着回流时间由 $15 \mathrm{~min}$ 延长至 $30 \mathrm{~min}$, 提取效率显著提 高, 继续延长至 $60 \mathrm{~min}$, 提取效率的提高减缓. 因此, 综 合考虑提取效率、试剂和时间成本等能效, 碳酸钻量 定为 $1 \mathrm{~g}$, 回流时间定为 $30 \mathrm{~min}$.

\section{4 方法的普适性}

使用标准品可以从理论上验证一种方法的可行 性, 但并不代表能用于实际检测, 主要原因在于实际 样本的基质复杂, 且不同来源的样本基质存在显著差 异(如动物蛋白、动物脂肪、植物和淀粉等), 在没有 合适的前处理过程配合下,往往无法实现实际样本的 检出. 因此, 在国标等各种应用于实际体系的检测标 准中, 在指明前处理流程下规定了可检测范围, 即方 法的普适性. 为了验证本文所提出的SERS方法检测 实际样本中二氧化硫的普适性, 采用同种前处理方法, 对不同来源的食品样本进行加标测试, 所得结果如图4 所示.

图4给出粉丝、生姜、金针菇、鱼片和冰糖等基 质中加标 $50 \mathrm{mg} \mathrm{kg}^{-1}$ 亚硫酸钠后, 经前处理得到的提取

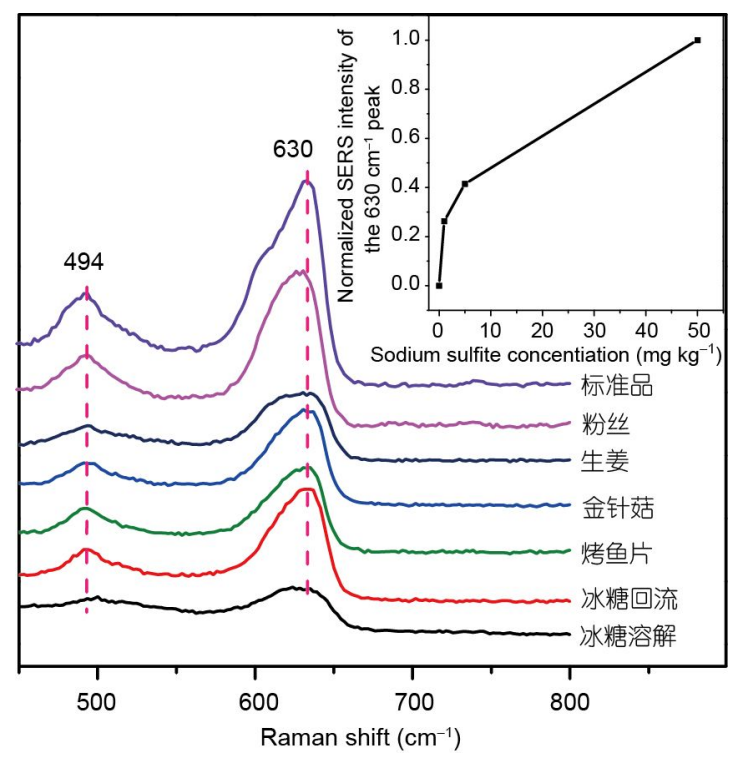

图 4 加标 $50 \mathrm{mg} \mathrm{kg}^{-1}$ 亚硫酸钠的不同食品样品, 经同样前处 理后得到的SERS谱图, 插图为以鱼干为例, 位于 $630 \mathrm{~cm}^{-1}$ 亚 硫酸钠特征峰的归一化强度随亚硫酸钠加标浓度的变化趋 势(网络版彩图)
液的SERS谱图. 根据图4可以获得 3 点信息. (1) 本研 究所改良的蒸馏法适用于不同基质的食品样本, SERS 方法对有无亚硫酸根的判断能力不受基质的影响, 定 性能力强. (2) 由于并未针对不同基质进行前处理优 化, 同一浓度的加标样本所得到SERS 谱峰的强度存 在一定差异, 信号波动可达 $50 \%$. 因此, 如果需要对 特定样本进行定量检测, 则必须针对性地考察样本回 收率, 进而绘制标准曲线. 例如, 插图所示鱼片样本 的浓度曲线: 在0 50 mg kg-1范围内, $\sim 630 \mathrm{~cm}^{-1}$ 谱峰的 SERS强度与亚硫酸加标浓度间存在正向定量关系, 说 明方法具有一定的定量可靠性. 此外, 相比于其他传 统方法, SERS方法的灵敏度高(最低检出浓度低)且无 假阳性. 需指出, 相比于图 1插图中 $0.1 \mathrm{mg} \mathrm{kg}^{-1}$ 的标准 品最低检出浓度, 实际样本的最低检出浓度提高到了 $1 \mathrm{mg} \mathrm{kg}^{-1}$, 主要是由于前处理过程不可避免地造成待 测物损失所致. 本研究建立的SERS方法的核心在于 快速便捷、高灵敏且普适性广, 因此, 未针对某一种样 品进行定向优化导致了提取回收率方面的不可避免 的损失. (3) 冰糖样本蒸馏或直接溶解后检测的结果 比对表明, 对于冰糖等基质较为简单的样本, 可以不 用蒸馏法进行前处理, 而只需简单的溶解, 即可对溶 液进行测试. 两种方案得到的SERS 谱图在强度上存 在一定的差异, 主要原因有两点: (1) 冰糖基质在SERS 增强基底上的竞争吸附, 导致了亚硫酸根SERS信号的 减弱; (2) 如图2所示, 蒸馏法的提取效率弱于直接检 测. 因此, 二者的共同效应导致直接检测方案得到的 SERS 强度偏弱. 对于土豆等通过表皮熏硫磺的固体 样本, 也可将SERS增强试剂直接滴加于样本表面进行 直接检测, 无需任何前处理.

\section{4 结论}

综上可知, 相比于间接检测二氧化硫类食品添加 剂的经典方法, 本研究利用SERS的指纹图谱的高能量 分辨率, 以亚硫酸根的特征拉曼谱峰为定性和定量依 据, 成功实现了对二氧化硫类物质(亚硫酸根、焦亚硫 酸根)的高灵敏的准确定性和半定量的直接检测. 同 时, 摒弃了传统蒸馏法使用的挥发性强酸和引入二次 污染的含铅吸收液, 改为环境友好的绿色试剂且不影 响目标物的检出. SERS方法普适性广, 操作简单, 具 有实际应用价值, 可用于常见食品中二氧化硫类滥用 和违禁使用的快速鉴定和篮查. 


\section{参考文献}

1 Kawamura Y, Satou T, Kubo N, Akano H, Kurosawa H, Nakamura K, Amano Y, Ito Y, Fujimaki M. J Food Sci Technol, 1992, 39: 233-238

2 许汉英, 王柯敏, 王大宁, 李庆才. 高等学校化学学报, 1998, 19: 1401-1404

3 马隽, 王兴华, 李宝华, 黄峰, 谢菲, 于爱民. 高等学校化学学报, 2006, 27: 39-42

4 Isaac A, Davis J, Livingstone C, Wain AJ, Compton RG. TrAC Trends Anal Chem, 2006, 25: 589-598

5 Wang S, Cui X, Fang G. Food Chem, 2007, 103: 1487-1493

6 Ruiz-Capillas C, Jiménez-Colmenero F. Food Chem, 2009, 112: 487-493

7 Li P, Wang X, Allinson G, Li X, Stagnitti F, Murray F, Xiong X. Environ Sci Pollut Res, 2011, 18: 1090-1097

8 Zhong Z, Li G, Zhu B, Luo Z, Huang L, Wu X. Food Chem, 2012, 131: 1044-1050

9 Wang X, Zhao W, Li L, Wang X, Li P, Wang Y, Luo J. Anal Methods, 2015, 7: 1036-1045

10 Deng Z, Chen X, Wang Y, Fang E, Zhang Z, Chen X. Anal Chem, 2015, 87: 633-640

11 祖文川, 汪雨, 张裕祥, 李冰宁, 刘聪, 任敏. 光谱学与光谱分析, 2016, 36: 1221-1224

12 Chen M, Yang H, Rong L, Chen X. Analyst, 2016, 141: 5511-5519

13 Frens G. Nat Phys Sci, 1973, 241: 20-22

14 Kurokawa Y, Imai Y, Sasaki Y, Maeda T. Anal Biochem, 1993, 209: 247-250

15 Damian Risberg E, Eriksson L, Mink J, Pettersson LGM, Skripkin MY, Sandström M. Inorg Chem, 2007, 46: 8332-8348

16 Müller IA, Brunner B, Breuer C, Coleman M, Bach W. Geochim Cosmochim Acta, 2013, 120: 562-581 


\title{
Developing fast laboratory screening platform for sulfate dioxide in food by surface-enhanced Raman spectroscopy
}

\author{
Lin Zhang ${ }^{1}$, Yongming Zeng ${ }^{2,3}$, Jianjun Zhao ${ }^{1}$, Hongju Chen ${ }^{4}$, Jinglin Kong ${ }^{1}$, Qizhen Chen ${ }^{2,3}$, \\ Huizhen Lin', Zhongqun Tian ${ }^{4}$, Guokun $\mathrm{Liu}^{2 *}$ \\ ${ }^{1}$ Stste Key Laboratory of NBC Protection for Civilian, Beijing 102205, China \\ ${ }^{2}$ State Key Laboratory of Marine Environmental Science, Department of the Environment \& Ecology, Xiamen University, Xiamen 361102 , China \\ ${ }^{3}$ PERSer Nanotechnology LTD., Xiamen 361021, China \\ ${ }^{4}$ Department of Chemistry \& Chemical Engineering, Xiamen University, Xiamen 361005, China \\ *Corresponding author (email: guokunliu@xmu.edu.cn)
}

\begin{abstract}
The direct detection of sulphur dioxide has been realized in various food matrixes with high sensitivity and high selectivity, on the basis of the integration of the high energy resolution of the finger-print spectrum of surface-enhanced Raman spectroscopy and the universal but easy-on-going pretreatment procedure. The characteristic peak of sulphur dioxide at $\sim 630 \mathrm{~cm}^{-1}$ was applied as the qualitative and quantitative standard, which displayed a lowest detectable concentration at the $1 \mathrm{mg} \mathrm{kg}^{-1}$ level for the spiked food samples. The key point of the high sensitivity and selectivity is the effective pretreatment born out of the standard distillation one, which has been improved in the three parts. (1) Using $\mathrm{CaCO}_{3}$ and Zeolite instead of $\mathrm{N}_{2}$ gas as the bubbling reagent. (2) Using oxalic acid as the acidic distillation solution to eliminate the hazards from acid volatilization, such as hydrochloric acid. (3) Using diluted sodium hydroxide solution as the absorption reagent instead of lead acetate solution to avoid the secondary pollution. With the three distinguished advantages of environment friendly, high sensitivity and free of matrix interference, the proposed method has great potential to replace the traditional ones for the fast screening of the illegal or abused sulphur dioxide in food.
\end{abstract}

Keywords: surface-enhanced Raman spectroscopy, quick-screening, environment friendly, high sensitivity

doi: $10.1360 / \mathrm{N} 032016-00197$ 